\title{
THE GROUP OF MEASURE PRESERVING TRANSFORMATIONS OF THE UNIT INTERVAL IS AN ABSOLUTE RETRACT
}

\author{
NGUYEN TO NHU \\ (Communicated by James E. West)
}

\begin{abstract}
The group of measure preserving transformations of the unit interval equipped with the weak topology is an absolute retract, hence is homeomorphic to a separable Hilbert space.
\end{abstract}

\section{INTRODUCTION}

Let $H$ denote the group of all measurable bijections on the unit interval $I=[0,1]$ preserving the Lebesgue measure. We identity $f \equiv g$ iff

$$
|\{t \in[0,1]: f(t) \neq g(t)\}|=0 .
$$

Here $|A|$ denotes the Lebesgue measure of $A$ in $I$.

The group $H$ will be endowed with the weak topology, that is, the topology induced by the family of pseudometrics

$$
\left\{d_{A}: A \text { is a measurable subset of } I\right\},
$$

where $d_{A}(f, g)=|f(A) \Delta g(A)|$ for $f, g \in H$.

Several topological properties of the group $H$ have been discovered. For instance, it is shown that $H$ is a separable complete metrizable group $[\mathrm{H}]$ and is contractible $[\mathrm{K}, \mathrm{D}]$. Let $H^{*}$ denote the subgroup of $H$ consisting of all piecewise continuous transformations. Dobrowolski [D] proved that if $H$ is an AR then $\left(H, H^{*}\right)$ is homeomorphic to $\left(\ell_{2}, \ell_{2}^{f}\right)$; here $\ell_{2}$ denotes the Hilbert space of all square summable sequences of real numbers and

$$
\ell_{2}^{f}=\left\{x=\left(x_{n}\right) \in \ell_{2}: x_{n}=0 \text { for almost all } n \in \mathbf{N}\right\} .
$$

Dobrowolski [D] conjectured that $H$ is an AR. The purpose of this note is to show that this conjecture is true. Namely, we are going to establish the following

Received by the editors March 21, 1989 and, in revised forms, May 1, 1989 and October 2, 1989; presented to the Summer Topological Conference, Oxford, England on June 28, 1989.

1980 Mathematics Subject Classification (1985 Revision). Primary 58D15, 58B05, 57N20, 54C55; Secondary 28D05.

Key words and phrases. Measure preserving transformations, absolute retract, Hilbert space. 
1.1. Theorem. $H$ is an $A R$.

As shown by Dobrowolski [D] our result provides a complete description of the topological property of $H$.

The idea of the proof of Theorem 1.1 is taken from $[\mathrm{N}]$, where it was shown that the space $M(X)$ of all measurable functions from $I$ into a metric space $X$ equipped with the topology of convergence in measure is an AR for any metric space $X$. As in $[\mathrm{N}]$ the proof of Theorem 1.1 is based on a characterization of ANR-spaces established by the author [N]. To apply the ANR-space characterization [N] we shall establish in $\S 2$ several auxiliary lemmas, notation, and conventions. This is the first step toward the proof of the main result. The proof of Theorem 1.1 is given in $\S 3$. In fact the result we prove in $\S 3$ is somewhat more general than Theorem 1.1. As a consequence, we also find a subgroup $G$ of $H$ for which $(H, G)$ is homeomorphic to $\left(\ell_{2}, \ell_{2}^{f}\right)$.

\section{LEMMAS, CONVENTIONS AND NOTATION}

In this section we shall establish several simple facts and introduce some notation and conventions which will be used later. First we show

2.1. Lemma. Let $A, B$ be measurable subsets of $I$. Then

(i) $\left|d_{A}-d_{B}\right| \leq 2|A \Delta B|$;

(ii) $d_{A \cup B} \leq d_{A}+d_{B}$.

Proof. For every $f, g \in H$ we have

$$
\begin{aligned}
d_{A}(f, g) & =|f(A) \Delta g(A)| \\
& \leq|f(A) \Delta f(B)|+|f(B) \Delta g(B)|+|g(B) \Delta g(A)| \\
& =d_{B}(f, g)+2|A \Delta B|,
\end{aligned}
$$

hence proving (i).

To prove (ii) we observe

$$
\begin{aligned}
d_{A \cup B}(f, g)= & |f(A \cup B) \Delta g(A \cup B)| \\
= & |f(A \cup B)|+|g(A \cup B)|-2|f(A \cup B) \cap g(A \cup B)| \\
\leq & 2|A \cup B|-2|f(A) \cap g(A) \cup f(B) \cap g(B)| \\
\leq & 2|A|+2|B|-2|A \cap B|-2|f(A) \cap g(A)| \\
& -2|f(B) \cap g(B)|+2|f(A) \cap f(B)| \\
= & d_{A}(f, g)+d_{B}(f, g) .
\end{aligned}
$$

The lemma is demonstrated.

To pave the way for the proof of Theorem 1.1 we shall introduce some notation and conventions.

If $\pi=\left\{a_{i}\right\}_{i=0}^{n}$ is a partition of $I$ we always assume that $a_{0}=0<a_{1}<\cdots<$ $a_{n}=1$. If $\pi_{1}=\left\{a_{i}\right\}_{i=0}^{n}$ and $\pi_{2}=\left\{b_{i}\right\}_{i=0}^{m}$ are partitions of $I$ then we denote 
$\pi_{1} \vee \pi_{2}=\left\{c_{i}\right\}_{i=1}^{k}$, where

$$
\left\{c_{i}\right\}_{i=1}^{k}=\left\{a_{i}\right\}_{i=1}^{n} \cup\left\{b_{i}\right\}_{i=1}^{m} .
$$

Therefore, $k \leq n+m$.

A function $f:[a, b) \rightarrow \mathbf{R}$ is a standard function iff there is a constant $c$ such that

$$
f(x)=x+c \quad \text { for every } x \in[a, b) .
$$

We say that $f \in H$ is a simple transformation iff there is a partition $\pi=$ $\left\{a_{i}\right\}_{i=0}^{n}$ such that $\left.f\right|_{\left[a_{i}, a_{i+1}\right)}$ is a standard function for every $i=0, \ldots, n-1$. In this case we write $f \perp \pi$ and we denote $f \perp \pi$ iff $f \perp \pi$ and $f^{-1} \perp \pi$.

We use the following notation:

$$
G=\text { the subgroup of } H \text { consisting of all simple transformations. }
$$

For every $f \in G$ we denote

$$
\pi(f)=\text { the smallest partition of } I \text { such that } f \perp \pi(f) .
$$

We need the following lemma.

2.2. Lemma. $G$ is dense in $H$.

Proof. Given $f \in H, \varepsilon>0$, and a measurable set $A \subset I$, we shall show that there exists $g \in G$ such that $d_{A}(f, g)<\varepsilon$.

To begin, we take disjoint intervals $\left[a_{i}, b_{i}\right)$ and $\left[c_{j}, d_{j}\right), i=1, \ldots, n$ and $j=1, \ldots, m$, satisfying the following conditions:

$$
\begin{gathered}
\left|A \Delta \bigcup_{i=1}^{n}\left[a_{i}, b_{i}\right)\right|<\frac{1}{2} \varepsilon ; \\
\left|f(A) \Delta \bigcup_{j=1}^{m}\left[c_{j}, d_{j}\right)\right|<\frac{1}{2} \varepsilon ; \\
\left|\bigcup_{i=1}^{n}\left[a_{i}, b_{i}\right)\right|=\left|\bigcup_{j=1}^{m}\left[c_{j}, d_{j}\right)\right| .
\end{gathered}
$$

We let $g \in G$ be any simple transformation such that

$$
g\left(\bigcup_{i=1}^{n}\left[a_{i}, b_{i}\right)\right)=\bigcup_{j=1}^{m}\left[c_{j}, d_{j}\right) \text {. }
$$

Denoting $B=\bigcup_{i=1}^{n}\left[a_{i}, b_{i}\right)$, we have

$$
\begin{aligned}
d_{A}(f, g) & =|f(A) \Delta g(A)| \\
& \leq|g(A) \Delta g(B)|+|g(B) \Delta f(A)| \\
& <|A \Delta B|+\frac{1}{2} \varepsilon<\varepsilon .
\end{aligned}
$$

This proves the lemma. 
Let $\pi=\left\{a_{i}\right\}_{i=0}^{n}$ be a partition of $I$. We write $A \prec \pi$ iff $A$ is a union of finite intervals of the form $\left[a_{i}, a_{i+1}\right), i \in\{0, \ldots, n-1\}$. If $f \in G$ we write $f \perp A$ iff $\left.f\right|_{\left(a_{i}, a_{i+1}\right)}$ and $\left.f^{-1}\right|_{\left[a_{i}, a_{i+1}\right)}$ are standard functions for every $\left[a_{i}, a_{i+1}\right) \subset A$. (Note that $A \prec \pi=\left\{a_{i}\right\}_{i=0}^{n}$.)

Given $f, g \in G$ such that $f \perp \pi$ and $g \perp \pi$. For every $\alpha \in[0,1]$ we define $\pi_{\alpha}(f, g)$ by the formula

$$
\pi_{\alpha}(f, g)(t)= \begin{cases}f(t) & \text { if } t \in\left[a_{i}, a_{i}+\alpha\left[a_{i+1}-a_{i}\right)\right) \\ g(t) & \text { if } t \in\left[a_{i}+\alpha\left(a_{i+1}-a_{i}\right), a_{i+1}\right) .\end{cases}
$$

The following lemma will be needed in the proof of Theorem 1.1.

2.3. Lemma. Assume that $f \perp \pi$ and $g \perp \pi$. Then

(i) $\pi_{\alpha}(f, g) \in G$ for every $\alpha \in[0,1]$;

(ii) If $A \prec \pi$ and $h \perp A$ then

$$
d_{A}\left(h, \pi_{\alpha}(f, g)\right) \leq \alpha d_{A}(h, f)+(1-\alpha) d_{A}(h, g) .
$$

The straightforward proof of Lemma 2.3 is left to the reader.

2.4. Remark. Let us note that Lemma 2.3 does not hold true without the assumptions that $f \perp \pi, g \perp \pi, h \perp A$, and $A \prec \pi$.

To apply the ANR-space characterization [N] for proving Theorem 1.1 we shall introduce a compatible metric $d$ for the group $H$.

For every $n \in \mathbf{N}$ we denote

$$
d_{n}(f, g)=\sum_{i=0}^{2^{n}-1} d_{\omega(n, i)}(f, g) \quad \text { for } f, g \in H,
$$

where $\omega(n, i)=\left[i 2^{-n},(i+1) 2^{-n}\right)$ for $i=0, \ldots, 2^{n}-1$.

Obviously $d_{n}(f, g) \leq 2$ for every $f, g \in H$.

From Lemma 2.1 it follows that $d_{n} \leq d_{m}$ for every $n \leq m$. We put

$$
d(f, g)=\sum_{n=1}^{\infty} 2^{-n} d_{n}(f, g) \quad \text { for } f, g \in H .
$$

Clearly $d$ is a metric on $H$. We show

2.5. Lemma. $d$ is a compatible metric on $H$.

Proof. It suffices to show that if $d_{n}\left(f_{k}, f\right) \rightarrow 0$ as $k \rightarrow \infty$ for every $n \in \mathbf{N}$, then $d_{A}\left(f_{k}, f\right) \rightarrow 0$ for every measurable set $A \subset I$.

Given $\varepsilon>0$, we select $B_{i} \subset I, i=1, \ldots, m$, such that:

$B_{i}$ is of the form $\left[n_{i} 2^{-n},\left(n_{i}+1\right) 2^{-n}\right)$ for $i=1, \ldots, m$ where $n_{i} \in\left\{0, \ldots, 2^{n}-1\right\}$;

$n$ is chosen so large that $|A \Delta B|<\frac{1}{4} \varepsilon$, where $B=\bigcup_{i=1}^{m} B_{i}$. 
Since $d_{n}\left(f_{k}, f\right) \rightarrow 0$ as $k \rightarrow \infty$ there is a $k_{0} \in \mathbf{N}$ such that $d_{n}\left(f_{k}, f\right)<\frac{1}{2} \varepsilon$ for every $k \geq k_{0}$. Whence from (3) and from Lemmas 2.1 and 2.2 we get

$$
\begin{aligned}
d_{A}\left(f_{k}, f\right) & \leq \frac{1}{2} \varepsilon+d_{B}\left(f_{k}, f\right) \\
& \leq \frac{1}{2} \varepsilon+d_{n}\left(f_{k}, f\right)<\varepsilon
\end{aligned}
$$

for every $k \geq k_{0}$. The lemma is proved.

In the sequence we shall consider the group $H$ with the metric $d$ defined by (1). The proof of Theorem 1.1 is based on the following characterization of ANR-spaces.

Let $\left\{\mathscr{U}_{n}\right\}$ be a sequence of open covers of a metric space $X$ and let $\mathscr{U}=$ $\bigcup_{n=1}^{\infty} \mathscr{U}_{n}$. By $\mathscr{N}(\mathscr{U})$ we denote the nerve of $\mathscr{U}$. We write $K \prec\left\{\mathscr{U}_{n}\right\}$ iff $K$ is a subcomplex of $\mathscr{N}(\mathscr{U})$ and every simplex $\sigma \in K$ is contained in $\mathscr{U}_{n} \cup \mathscr{U}_{n+1}$ for some $n \in \mathbf{N}$. We let

$$
n(\sigma)=\max \left\{n: \sigma \subset \mathscr{U}_{n} \cup \mathscr{U}_{n+1}\right\} .
$$

We shall use the following

2.6. Theorem [N]. A separable metric space $X \in A N R$ if and only if there exists a sequence of open covers $\mathscr{U}_{n}$ of $X$ such that for any locally finite simplicial complex $K \prec\left\{\mathscr{U}_{n}\right\}$ and for any selection $f: K^{0} \rightarrow X$ (i.e., $f(V) \in V$ for every $V \in K^{0}$ ) there is a map $g: K \rightarrow X$ such that for any sequence $\left\{\sigma_{k}\right\}$ of simplices of $K$ with $n\left(\sigma_{k}\right) \rightarrow \infty$ as $k \rightarrow \infty$ we have

$$
\delta\left(\sigma_{k}\right)=\sup \left\{d(f(V), g(x)): V \in \sigma_{k}^{0}, x \in \sigma_{k}\right\} \rightarrow 0 .
$$

\section{Proof of The MAIN Result}

Now we are already in a position to prove Theorem 1.1. We shall establish the following more general result.

3.1. Theorem. $\widetilde{G}$ is an ANR for any subset $\widetilde{G}$ of $H$ containing $G$.

Since $H$ and $G$ are contractible, see [D], from Theorem 3.1 and from the proof of [D, Theorem 2] of Dobrowolski, we obtain

3.2. Corollary. $(H, G)$ is homeomorphic to $\left(\ell_{2}, \ell_{2}^{f}\right)$.

Related to Corollary 3.2 we ask

3.3. Question. Is there a subgroup $S$ of $H$ such that $(H, S, G) \cong\left(l_{2}, l_{2}^{\sigma}, l_{2}^{\sigma}\right)$ or $\left(H, S, H^{*}\right) \cong\left(l_{2}, l_{2}^{\sigma}, l_{2}^{f}\right)$ where $l_{2}^{\sigma}=\left\{x=\left(x_{n}\right) \in l_{2}: \sum_{n=1}^{\infty}\left(n x_{n}\right)^{2}<\infty\right\}$ ?

Proof of Theorem 3.1. We shall show that $\tilde{G}$ satisfies the condition of Theorem 2.6.

Take a sequence $\left\{\mathscr{U}_{n}\right\}$ of open covers of $\widetilde{G}$ such that $\operatorname{diam} V<2^{-n}$ for every $V \in \mathscr{U}_{n}$. Let $K \prec\left[\left\{\mathscr{U}_{n}\right\}\right.$ be a locally finite simplicial complex and let 
$f: K^{0} \rightarrow \widetilde{G}$ be a selection. Using Lemma 2.2 we can choose a map $g_{0}: K^{0} \rightarrow G$ such that

$$
d\left(f(V), g_{0}(V)\right)<2^{-n(V)} \text { for every } V \in K^{0},
$$

where $n(V)=\max \left\{n: V \in \mathscr{U}_{n}\right\}$.

For each simplex $\sigma \in K$ let $A(\sigma)$ denote the set of all vertices of simplices of $K$ containing $\sigma$ as a face. From the local finiteness of $K$ it follows that $A(\sigma)$ is finite for every $\sigma \in K$.

We shall define inductively a sequence of maps $g_{k}: K^{(k)} \rightarrow G$ with the following properties:

$$
g_{k} \mid K^{(k-1)}=g_{k-1} \quad \text { for every } k \geq 1 ;
$$

(6) for every $\sigma \in K^{(k)}$ there is an $n \in \mathbf{N}$ such that $g_{k}(\sigma \cup A(\sigma)) \subset$ $G_{n}$, where

$$
\begin{array}{r}
G_{n}=\left\{f \in G: \text { there exits a partition } \pi=\left\{a_{i}\right\}_{i=0}^{m}\right. \\
\text { of } I \text { with } m \leq n \text { such that } f \perp \pi\} ;
\end{array}
$$

(7) for every $\sigma \in K^{(k)}$, for every $h \in g_{0}(A(\sigma))$ and for every $x \in \sigma$ we have

$$
d\left(h, g_{k}(x)\right) \leq \max \left\{d\left(h, g_{0}(V)\right): V \in \sigma^{0}\right\}+\left(1-2^{-k}\right) \operatorname{diam} g_{0}\left(\sigma^{0}\right) .
$$

Clearly $g_{0}$ satisfies conditions (6) and (7). Assume that $g_{k-1}$ has been defined with the properties (5)-(7). We define $g_{k}: K^{(k)} \rightarrow G$ as follows: For each $\sigma \in K^{(k)}$ let $c$ be an interior point of $\sigma$. We put $g_{k}(c)=g_{0}(V)$, where $V$ is a vertex of $\sigma$. Note that for every $x \in \sigma, x \neq c$, there exists a unique $\alpha \in[0,1]$ and $y \in \dot{\sigma}$ (the boundary of $\sigma$ ) such that $x=\alpha c+(1-\alpha) y$. By the inductive assumption there is an $n_{0} \in \mathbf{N}$ such that

$$
\bigcup\left\{g_{k-1}\left(\sigma^{\prime} \cup A\left(\sigma^{\prime}\right)\right): \sigma \text { is a face of } \sigma\right\} \subset G_{n_{0}} .
$$

Choose $m>n_{0}$ such that

$$
n_{0}(\operatorname{Card} A(\sigma)+1) 2^{-m}<2^{-k-5} \operatorname{diam} g_{0}\left(\sigma^{0}\right) \text {. }
$$

Let $\pi=\pi_{m} \vee \pi\left(g_{k}(c)\right) \vee \pi\left(g_{k-1}(y)\right)$, where $\pi_{m}=\left\{i 2^{-m}\right\}_{i=0}^{2^{m}}$ and define $g_{k}(x)$ by the formula

$$
g_{k}(x)=\pi_{\alpha}\left(g_{k}(c), g_{k-1}(y)\right) .
$$

It is easy to see that $g_{k}$ is continuous.

Obviously $g_{k}$ satisfies conditions (5) and (6). We shall verify (7). Denote

$$
\omega=\bigcup\left\{\omega(m, i) \subset I:\left.g_{k-1}(z)\right|_{\omega(m, i)} \quad \text { or }\left.\quad g_{k-1}^{-1}(z)\right|_{\omega(m, i)}\right.
$$

is not a standard function for some $z \in A(\sigma) \cup\{y\}\}$.

(Recall that $\omega(n, i)=\left[i 2^{-m},(i+1) 2^{-m}\right)$.) 
For every $n \leq m$ and for every $i=0, \ldots, 2^{n}-1$, from Lemma 2.1 we get

$$
\begin{aligned}
d_{n}\left(h, g_{k}(x)\right)= & \sum_{i=0}^{2^{n}-1} d_{\omega(n, i)}\left(h, g_{k}(x)\right) \\
\leq & \sum_{i=0}^{2^{n}-1} d_{\omega(n, i) \backslash \omega}\left(h, g_{k}(x)\right) \\
& +\sum_{i=0}^{2^{n}-1} d_{\omega(n, i) \cap \omega}\left(h, g_{k}(x)\right) .
\end{aligned}
$$

Observe that

$$
\sum_{i=0}^{2^{n}-1} d_{\omega(n, i) \cap \omega}\left(h, g_{k}(x)\right) \leq 2|\omega| .
$$

From Lemmas 2.1 and 2.3, we have

$$
\begin{aligned}
& \sum_{i=0}^{2^{n}-1} d_{\omega(n, i) \backslash \omega}\left(h, g_{k}(x)\right) \\
& \quad \leq \sum_{i=0}^{2^{n}-1}\left(\alpha d_{\omega(n, i) \backslash \omega}\left(h, g_{k}(c)\right)+(1-\alpha) d_{(n, i) \backslash \omega}\left(h, g_{k-1}(y)\right)\right. \\
& \quad \leq \alpha d_{n}\left(h, g_{k}(c)\right)+(1-\alpha) d_{n}\left(h, g_{k-1}(y)\right)+2|\omega|
\end{aligned}
$$

Hence

$$
\begin{aligned}
d\left(h, g_{k}(x)\right)= & \sum_{n=1}^{\infty} 2^{-n} d_{n}\left(h, g_{k}(x)\right) \\
= & \sum_{n=1}^{m} 2^{-n} d_{n}\left(h, g_{k}(x)\right)+\sum_{n=m+1}^{\infty} 2^{-n} d_{n}\left(h, g_{k}(x)\right) \\
\leq & \sum_{n=1}^{m} 2^{-n}\left(\alpha d_{n}\left(h, g_{k}(c)\right)+(1-\alpha) d_{n}\left(h, g_{k}(y)\right)\right) \\
& +4|\omega|+2^{-m+1} \\
\leq & \alpha d\left(h, g_{k}(c)\right)+(1-\alpha) d\left(h, g_{k-1}(y)\right)+4|\omega|+2^{-m+1} .
\end{aligned}
$$

From (8) and (9) we get

$$
4|\omega|+2^{-m+1}<2^{-k} \operatorname{diam} g_{0}\left(\sigma^{0}\right) .
$$

Let $\sigma^{\prime} \in K^{(k-1)}$ denote a face of $\sigma$ containing $y$. Then, by inductive assumption, for every $h \in g_{0}\left(A\left(\sigma^{\prime}\right)\right)$ we have

$$
\begin{aligned}
d\left(h, g_{k-1}(y)\right) & \leq \max \left\{d\left(h, g_{0}(V)\right): V \in \sigma^{\prime 0}\right\}+\left(1-2^{-k+1}\right) \operatorname{diam} g_{0}\left(\sigma^{\prime 0}\right) \\
& \leq \max \left\{d\left(h, g_{0}(V)\right): V \in \sigma^{0}\right\}+\left(1-2^{-k+1}\right) \operatorname{diam} g_{0}\left(\sigma^{0}\right) .
\end{aligned}
$$


Observe that $A\left(\sigma^{\prime}\right) \supset A(\sigma)$. Therefore, for every $h \in g_{0}(A(\sigma))$ we have

$$
\begin{aligned}
d\left(h, g_{k}(c)\right) \leq & \max \left\{d\left(h, g_{k}(c)\right), d\left(h, g_{k-1}(y)\right)\right\}+2^{-k} \operatorname{diam} g_{0}\left(\sigma^{0}\right) \\
\leq & \max \left\{d\left(h, g_{0}(V)\right): V \in \sigma^{0}\right\}+2^{-k} \operatorname{diam} g_{0}\left(\sigma^{0}\right) \\
& +\left(1-2^{-k+1}\right) \operatorname{diam} g_{0}\left(\sigma^{0}\right) \\
\leq & \max \left\{d\left(h, g_{0}(V)\right): V \in \sigma^{0}\right\}+\left(1-2^{k}\right) \operatorname{diam} g_{0}\left(\sigma^{0}\right)
\end{aligned}
$$

Consequently condition (7) holds. Finally we define $g: K \rightarrow G$ by the formula

$$
g(x)=\lim _{k \rightarrow \infty} g_{k}(x) \text { for every } x \in K .
$$

Condition (7) shows that $g$ satisfies the condition of Theorem 2.6. Therefore $\widetilde{G} \in A N R$.

This completes the proof of Theorem 3.1.

Since the unit interval $I$ with Lebesgue measure is measure isomorphic with any complete separable metric equipped with its Borel measure, Theorem 1.1 remains true if $I$ is replaced by any such metric space.

\section{REFERENCES}

[D] T. Dobrowolski, Examples of topological groups homeomorphic of $\ell_{2}^{f}$, Proc. Amer. Math. Soc. 98 (1986), 303-311.

[H] P. R. Halmos, Lectures on ergodic theory, Chelsea Publishing Company, New York, 1956.

[K] M. Keane, Contractibility of the automorphism group of a nonatomic measure space, Proc. Amer. Math. Soc. 26 (1970), 420-422.

[N] N. T. Nhu, Investigating the ANR-property of metric spaces, Fund. Math. 124 (1984), 243254.

Institute of Mathematics, Post Office Box 631, Bo Ho, Hanoi, Vietnam 